\title{
Curative Effects Observation of the Foot Care on Type 2 Diabetes Peripheral Neuropathy
}

\author{
Xiumei Zhang*, Kunzhu Huang and Yanshan Gu \\ Department of Endocrinology, The First Affiliated Hospital of Kunming Medical University, Kunming, \\ Yunnan 650032, China
}

\begin{abstract}
Objective: To discuss the curative effects of foot care on type 2 diabetes peripheral neuropathy (DPN). Methods: This study selected 80 cases of type 2 diabetes peripheral neuropathy, and divided them into two groups randomly: the experimental group and the control group, both of the two groups were treated with standard hypoglycemic therapy plus promoting blood circulation and removing blood stasis drugs and nutritional nerve medicine therapy and regular nursing, the observation group was conducted foot care, education and guidance on the basis of these measures in 3 months. Assessed the improvement of the double lower limbs symptoms and the speed of lower limbs sensory nerve conduction velocity. Results: The curative effects of the observation group were more significant than the control group $(p<0.01)$, the overall efficiency was $86 \%$, and the improvement of the lower limbs sensory nerve conduction velocity was superior to the control group $(p<0.01, p<0.05)$. Conclusion: The foot care can improve the microcirculation, promote the repair of the damaged nerve, improve the DPN symptoms, and prevent the occurrence of the diabetic foot.
\end{abstract}

\section{KEYWORDS}

Foot care

Type 2 diabetes peripheral neuropathy Curative effects

\section{Introduction}

According to the relevant documents in 2010 [1], he occurrence rate of diabetes in China is $9.7 \%$, the number of diabetes patients is almost 92.4 million, and the occurrence rate of diabetes is increasing year by year. Diabetes peripheral neuropathy diseases are the most common complication of diabetes, and the rate of patients with a disease history above 10 years exceeds 50\% [2]. There are various clinical symptoms, with the symmetrical lamb pains, sensory loss, numbness, burning or cold feeling as the principal symptoms, usually the lower limbs are more serious than the upper limbs. In the primary period, the main symptom is mainly sensory disturbance, and in the later period the patient can exhibit the symptom of motor nerve

Copyright (c) 2012 Xiumei Zhang et al.

doi: 10.18686/jn.vli1.2

Received: May 9, 2012; Accepted: August 26, 2012; Published online: October 28, 2012

This is an open-access article distributed under the terms of the Creative Commons Attribution Unported License (http://creativecommons.org/ licenses/by-nc/4.0/), which permits unrestricted use, distribution, and reproduction in any medium, provided the original work is properly cited.

${ }^{\star}$ Corresponding author: Department of Endocrinology, The First Affiliated Hospital of Kunming Medical University, Kunming, Yunnan 650032, China. E-mail: zxm_0093@163.com damage, which can both lead to the diabetes foot and affect the patients' life quality. In 2010-2011, this study selected 80 cases of type 2 DPN patients and conducted foot care on these patients, and received satisfactory results. Now it is reported as follows.

\section{Data and methods}

\subsection{General data}

This study selected 80 cases of patients with DPN in the outpatient between 2010 and 2011. Selective criteria: The diagnosis and type of the diabetes conform to the WTO diagnosis criteria [3], with different extent of peripheral neuropathy, such as limb acro-numbness, scorching hot, cold feeling, acupuncture pains, the feeling of socks, the feeling of foot pad, the formication or dysesthesia and so on. Exclude the patients who have foot ulcers or gangrene, the mental disorder or the retarded patients who cannot cooperate. These patients were divided into two groups randomly, 42 cases in the observation group, 38 cases in the control group. In the observation group, 22 males and 20 females, age ranges from $38-77$ years old, average age: 60.5; disease history $5-23$ years, 11.5 years at average; the patients took the hypoglycemic agents orally in 19 cases, received insulin therapy in 23 cases. In the control group, 20 males and 18 females, age ranges $39-78$ years old, aver- 
age age: 61.2 , disease history $4-24$ years, 11.8 years at average; the patients took the hypoglycemic agents orally in 17 cases, received insulin therapy in 21 cases. There was no difference in the statistics sense $(p>0.05)$ in the respect of gender, age, disease history and other general conditions, so the two groups have comparability.

\subsection{Methods}

\subsubsection{Intervention method}

The patients in two groups all received standard hypoglycemic therapy, and the blood sugar control reached the standard (Fasting blood sugar $<7.0 \mathrm{mmol} / \mathrm{L}$, blood sugar after the meal within 2 hours $<9.0 \mathrm{mmol} / \mathrm{L}$ ), plus the promoting blood circulation and removing blood stasis drugs and nutritional nerve medicine therapy. The control group was conducted standard nursing guidance, and the observation group received standard nursing plus foot care, and the diabetes professional nurses provided relevant guidance for the patients and their families, including the pathogeny of DPN, the serious effects on the patients of ineffective intervention or no-intervention, the importance of foot care and so on, and demonstrated the foot care methods, pushed them forward to master the skills, and required the families to implement the foot care.

\section{Promote blood circulation of the limbs}

Take leg exercises every day. (1) Lift the heel: Stand on the feet, stand straight, and lift the heel and put down, repeat 20 times. (2) Lift the tiptoe: Stand straight and lift, put down the tiptoe, repeat 20 times. (3) Swing the leg: Put one let on a brick with the hands on the chair, and swing the other leg forth and back, after 10 times put the tiptoe on the floor, and the ankle joint rotates 20 times in clockwise and anticlockwise. Then anther leg repeats the same actions stated above. (4) Bending: Bending forward with hands holding the chair, and the head should be as low as possible, and the back should be as straight as possible when bending, and repeat 10 times. (5) Chair-sitting exercise: Stick out the chest, sit straight with the arms crossing before the chest, sit down and stand up, repeat 10 times. (6) Counter exercise: Hands stretch out on the floor, the feet were put together with the heel on the floor, straight the body, place the gravity on the arms, and stretch out and bend the arms, repeat 10 times. The patients should take 2 groups of the entire exercises stated above.

\section{Foot massage}

The diabetes professional nurses provide guidance to the patients and their families. Put the feet into the water with the temperature of $37-40^{\circ} \mathrm{C}$ for $5-10 \mathrm{~min}$, and then wipe the feet with soft towels, especially the space between the toes. Examine the foot skins carefully, especially whether there are any bruises between the toes, the sole of the foot and other parts which are vulnerable to be squeezed. If there exists skin itching or ringworm of the foot, enjoin the patient not to scratch with hands. Put the feet into warm water and wipe them dry, then smear the feet with skin oil. The operator first hold one foot with the thumbs and other four fingers, press the entire sole of the foot slightly gradually from the tiptoe to the heel, and after a while change on the other foot. Then place the two hands on the instep and palm, rub the entire foot gently up and down to promote the blood circulation of the foot and then rub the toes with hands, and take the massage from the tiptoe up to the shank gradually. At last, rub the acupuncture point of Zusanli, Sanyinjiao, Yanglingquan, Taixi etc. [4]. The entire massage lasts $20-30 \mathrm{~min}$. Keep warm and wears loose shoes and socks.

\subsection{Curative effect evaluation criteria}

Take the evaluation of these two groups before the intervention and after the intervention within 3 months, and the curative effects are: significant (acro-numbness, pains, and fatigue were relieved significantly, and sleep well at night), effective (acro-numbness, pains, and fatigue were relieved slightly, and sleep at night improve slightly), ineffective (neither the symptoms or the sleep at night has improved). Sensory nerve conducting velocity (SNCV), use cantata TM type electromyogram presented by Danish company called Dandy to conduct nerve electrophysiological check, and measure the patients' SNCV of bilateral peroneal nerve and posterior tibial nerve. The SNCV of a healthy person's peroneal nerve is $50.26 \pm 6.62 \mathrm{~m} / \mathrm{s}$, posterior tibial nerve $56.76 \pm 5.52 \mathrm{~m} / \mathrm{s}$ [5]. Judgment on the nerve damage extent: (1) Slight: NCV slows down 15-30\%. (2) Medium: NCV slows down 30-40\%. (3) Serious: NCV slows down $>40 \%$ or cannot produce the nerve unit potential.

\subsection{Statistics methods}

Employ the SPSS 15.0 statistics software to analyze, apply $\chi^{2}$ test, $t$ test to analyze the data, and use $p<0.05$ to indicate that the difference is significant in statistic sense.

\section{Results}

\subsection{Comparison between the Two Groups}

The total effective rate of observation group is $86 \%$, which is significant higher than the control group $(p<0.01)$, see Table 1.

\subsection{Comparison of lower limbs SNCV measurement results}

Comparison of lower limbs SNCV measurement results before and after the treatment between the two groups, see table 2.

\section{Discussion}

With the increase of population aging and the occurrence of diabetes, the incidence of diabetic foot in China is also increasing. The occurrence rate of diabetic foot is 
Table 1. Comparison between the two groups.

\begin{tabular}{|c|c|c|c|c|c|c|c|c|}
\hline \multirow{2}{*}{ Group type } & \multirow{2}{*}{$n$} & \multicolumn{2}{|c|}{ Significant effective } & \multicolumn{2}{|c|}{ Effective } & \multicolumn{2}{|c|}{ Ineffective } & \multirow{2}{*}{$\begin{array}{c}\text { Total effective rate } \\
\%\end{array}$} \\
\hline & & $\mathrm{n}$ & $\%$ & $\mathrm{n}$ & $\%$ & $\mathrm{n}$ & $\%$ & \\
\hline Control group & 38 & 8 & 21 & 11 & 29 & 19 & 50 & 50 \\
\hline Observation group & 42 & 23 & 55 & 13 & 31 & 6 & 14 & $86^{\triangle}$ \\
\hline
\end{tabular}

Note: Compared to the control group, ${ }^{\Delta} p<0.01$.

Table 2. Comparison of lower limbs SNCV measurement results before and after treatment between the two groups (m/s, $\bar{x} \pm \mathrm{s}$ ).

\begin{tabular}{|c|c|c|c|c|}
\hline \multirow{2}{*}{ Project } & \multicolumn{2}{|c|}{ Control group $(n=38)$} & \multicolumn{2}{|c|}{ Observation Group ( $n=42$ ) } \\
\hline & Before the treatment & After the treatment & Before the treatment & After the treatment \\
\hline Peroneal nerve & $30.40 \pm 3.5$ & $33.6 \pm 3.6^{*}$ & $31.46 \pm 4.2$ & $38.60 \pm 4.6^{* *}$ \\
\hline Posterior tibial nerve & $36.21 \pm 2.7$ & $41.1 \pm 2.8^{*}$ & $37.21 \pm 2.9$ & $44.35 \pm 3.2^{* *}$ \\
\hline
\end{tabular}

Note: Compared to the control group before the treatment, ${ }^{*} p<0.05$, compared to the control group, ${ }^{* *} p<0.01$.

$60-70 \%$, and is resulted from DPN, so in clinical respect it is very difficult to cure, and it is the main reason for nontraumatic amputation, which can affect the patients' life quality seriously. So it is extremely important to prevent or delay the occurrence of DPN [6].The pathogenesis of DPN is currently considered to be mainly metabolic disorder and vascular disorder, and the symptoms are the function abnormality of the nerve nourish blood vessels and capillaries in the inner and outer membrane or blood flow abnormality, which can lead to the lack of blood and oxygen in the endometrium, and further affect the nerve cell's normal physiological metabolism, leading to chronic injury of nerve tissue, and the symptoms are nerve sheath injury and axonal degeneration [7]. Abnormal glucose metabolism can directly affect the nerve tissue. It can decrease the activity of $\mathrm{Na}^{+}-\mathrm{K}^{+}$-ATP enzyme [8], disturb the protein synthesis, make the lipid composition of the nerves and lipid composition of myelin sheath abnormal, and lead to nerve cell edema, axonal degeneration, the degradation of the physical functions, and the slowdown of the sensory speed.

On the basis of regular hypoglycemic therapy and nursing of the patients in 42 cases, this study provide health education and foot care guidance to the patients and their families in the control group, make them participate in the daily nursing activities positively, and judge the curative effects by the criteria of the patients' subjective feelings and objective check results (SNCV), and use the patients in 38 cases as comparison. Research results show: The curative effect of the control group is more significant than the control group $(p<0.01)$, total effective rate is $86 \%$, the results see Table 1. After treatment, the control group's nerve conductivity velocity quickens significantly, and the improvement of SNCV of peroneal nerve and posterior tibia nerve is more significant than the control group $(p<0.01, p<$ 0.05 ), and the damaged extent of the nerve is also relieved, see Table 2. Leg exercises can promote the limbs blood circulation, and lower the blood viscosity, angiotelectasis, provide sufficient blood for the feet, make the drug of promoting blood circulation and removing blood stasis arrive at and absorbed by the feet, and improve the nerve's status of lacking blood and oxygen, promote the repair of the nerve, and improve the physical metabolism, use the glucose and help to reduce the blood sugar. Foot massage can promote the feet's local angiotelectasis, increase the feet's blood supply, improve the feet's microcirculation and the status of lacking of blood and oxygen of feet, repair the damaged nerve, fasten the nerve conductivity speed, relieve the clinical symptoms, enhance the patients' life confidence, and life quality.

Research confirms, the implement on the patients of foot care plus medical therapy can promote the double lower limbs blood supply, extend the blood vessel, increase the body's blood supply, quicken the blood circulation speed, improve the microcirculation, and the nerve's status of lacking of oxygen, give the nerve enough nutrition, promote the repair of the damaged nerve, improve the symptoms of DPN, prevent the occurrence of diabetic feet. The curative effect of foot care combined with education is significant, and the short time of foot care can improve the patients' symptoms significantly, and symbolize the importance of nursing. Regular follow-ups, education and supervision can enhance the patients' dependence on the cooperative nursing, and the consciousness of self-nursing, strengthen the confidence of prevention and therapy, and receive good prevention, treatment and education effects.

\section{Conflicts of interest}

These authors have no conflicts of interest to declare.

\section{Authors' contributions}

These authors contributed equally to this work.

\section{References}

1. Yang W, Lu JM, Wing JP, et al. Prevalence of diabetes among men and women in China. $N$ Engle $J$ Med. 2010;362(12):1090-1101.

2. Song QF, Song GY. Analysis of risk factors for type 2 dia- 
betes peripheral neuropathy. Chinse Journal of Gerontology. 2009;29(2):222-224.

3. Lu ZY, Zhong NS. Internal Medicine. The $7^{\text {th }}$ Edition. Beijing: People's Medical Publishing House. 2008:770-793.

4. Zhang Chunling, Xie Jing, Chen Lu, etc. Dialectically nursing of 80 patients with diabetic feet. Journal of Nursing Science. 2009;24(5):53-54.

5. Wang HL, Yang MY, Wen XL. Application Value of Nerve Conduction Velocity Determination and F Wave Examination in DPN Diagnosis. Journal of Baotou Medical Coloege. 2008,24(2):146-147.
6. Chen Y, Li H, Shi Y. Effect of foot care on prognosis of patients with diabetes mellitus peripheral neuropathy disease. Journal of Nursing Science. 2009;24(5):34-35.

7. Zhang RF. Observation of curative effect of mecobalamin, defibrase combined clinical observation on treatment of diabetic neuropathy in 98 cases. Journal of Dalian Medical University. 2003;25(1):46-47.

8. Zhong MR, Fu XM, Fu WL, etc. Research progress of diabetic peripheral neuropathy pathogenesis and treatment of traditional Chinese medicine. Journal of Chengde Medical College. 2009;26(4):438-440. 